\title{
Nonlinear parameter retrieval from three- and four-wave mixing in metamaterials
}

\author{
Alec Rose, Stéphane Larouche, Da Huang, Ekaterina Poutrina, and David R. Smith \\ Department of Electrical and Computer Engineering, Center for Metamaterials and Integrated Plasmonics, Pratt School of Engineering, \\ Duke University, P.O. Box 90291, Durham, North Carolina 27708, USA
}

(Received 11 June 2010; published 28 September 2010)

\begin{abstract}
We present a generalized nonlinear susceptibility retrieval method for metamaterials based on transfer matrices and valid in the nondepleted pump approximation. We construct a general formalism to describe the transfer matrix method for nonlinear media and apply it to the processes of three- and four-wave mixing. The accuracy of this approach is verified via finite element simulations. The method is then reversed to give a set of equations for retrieving the nonlinear susceptibility. Finally, we apply the proposed retrieval operation to a three-wave mixing transmission experiment performed on a varactor loaded split ring resonator metamaterial sample and find quantitative agreement with an analytical effective medium theory model.
\end{abstract}

DOI: 10.1103/PhysRevE.82.036608

PACS number(s): 41.20.-q, 42.65.Ky, 78.67.Pt

\section{INTRODUCTION}

Metamaterials are artificially and purposefully structured materials whose electromagnetic responses are described by effective, homogenized constitutive parameters. Such materials have generated interest due to their ability to display properties that are either limited or entirely unachievable in naturally occurring materials [1-3]. Recently, many researchers have begun to investigate the effects of integrating nonlinear elements into metamaterials [4-9]. This research has been sparked, in part, by the observation that in many existing and proposed resonant metamaterials, the incident fields are found to have highly nonuniform distributions over a unit cell, leading to significant confinement of electromagnetic energy in small, critical volumes [1]. Many metamaterials, for example, are based on metal-patterned substrates that operate analogously to $R L C$ circuits, such as the split ring resonator (SRR) and the electric-field-coupled resonator (ELC). When these metamaterials are operated near their resonance frequencies, the electric field in the capacitive gaps is much stronger than the macroscopically averaged value. Thus, the nonlinear properties of any material placed into these critical volumes are enhanced considerably $[4,10]$. Additionally, at radio and microwave frequencies, it is possible to utilize nonlinear electronic components for this purpose [11-17]. The canonical example is the varactor loaded split ring resonator (VLSRR), formed by integrating a varactor diode into the capacitive gap of the SRR.

For the vast majority of metamaterials, a method of homogenization is necessary to describe the effective response of their engineered structure. This process of effective parameter retrieval is vital for the characterization of fabricated metamaterials, as well as for the design of potential metamaterials relevant to specific applications. In essence, the retrieval process consists of equating the unit cell of the metamaterial to a congruently sized homogeneous material with an unknown set of parameters. In the linear case, the system is commonly solved by finding the equivalent permittivity and permeability that replicate the scattering parameters of the metamaterial [18-20]. This retrieval method has the added advantage of being easily applicable in practice by implementing transmission and reflection experiments on a metamaterial sample. In order to effectively describe and design nonlinear metamaterials, a similar process is needed.

To this end, Larouche and Smith have recently proposed the use of a modified transfer matrix method for the retrieval of effective nonlinear susceptibilities in metamaterials [21]. The transfer matrix method for nonlinear media is described by Bethune for the calculation of third harmonic generation (THG) [22]. Larouche and Smith adapt the transfer matrix approach to the particular case of second harmonic generation (SHG), demonstrating that, for a layered system with known properties, the output harmonics can be accurately and efficiently computed from the incident fields in the nondepleted pump approximation - that is, under the assumption that higher-order harmonics do not perturb the field pattern of the fundamental mode. The transfer matrix method can then be reversed to perform the opposite operation, in which the output harmonics, determined from simulation or experiment, are used to retrieve an effective nonlinear susceptibility. The usefulness of this method has been recently demonstrated at microwave frequencies using a VLSRR medium [23], where excellent agreement between the measured and theoretically predicted properties of a VLSRR medium was found.

The characterization of harmonics such as SHG or THG for nonlinear metamaterials represents only a subset of the applicability of nonlinear retrieval methods to metamaterials. In this paper, we extend the transfer matrix method formalism to incorporate an arbitrary-order nonlinearity and arbitrary input waves. We then explicitly apply the method to three- and four-wave mixing processes, validating the determined field distributions of the sum and difference modes via finite element time-domain simulations. The extended transfer matrix method is then reversed, and the generalized nonlinear retrieval operation is demonstrated in the analysis of a three-wave mixing transmission experiment performed on a VLSRR sample.

\section{THEORY}

The configuration of numerous recent nonlinear metamaterial experimental and theoretical studies has been a onedimensional system composed of layered slabs, in which at 
least one layer possesses a significant nonlinear susceptibility. A reasonable goal, given such a system, is to determine the steady-state complex field amplitudes at all positions for a given set of incident waves. The presence of the nonlinearity precludes the use of most conventional methods of solution, including transfer matrices, as these methods rely on the linear properties of a system. However, in many such experiments the nonlinear processes are weak enough that their effect on the incident waves is negligible, leaving the fields at these frequencies nearly identical with those expected in a linear system. This is known as the nondepleted pump (NDP) approximation, and, in this limit, an exact method of evaluation can be formulated. Hence, the NDP approximation is assumed in the following analysis. In this section, we outline a general formalism for the modified transfer matrix method, apply it to three- and four-wave mixing, and present the resulting nonlinear retrieval equations. The following analysis assumes an electric nonlinearity, but can be carried through identically for a magnetic nonlinearity, replacing references to the electric field with the magnetizing field, the polarization with the magnetization, and swapping all occurrences of the permittivity and the permeability.

\section{A. Overview of the transfer matrix method}

In essence, the transfer matrix method for nonlinear processes involves three steps [22]. First, the incident waves are linearly propagated by the usual transfer matrix operations, giving the electric fields at the fundamental frequency(ies) everywhere in the system. Second, the fields are used to calculate the material's nonlinear polarization, which in turn can be treated as a field-generating source term. Finally, the fields thus created are propagated via transfer matrices to both boundaries of the system, yielding the reflected and transmitted field amplitudes at the generated frequencies. The system under consideration in this paper is presented in Fig. 1 .

\section{For linear materials}

To demonstrate the transfer matrix approach, we consider a uniform slab of thickness $d$, permittivity $\varepsilon_{2}(\omega)$, and permeability $\mu_{2}(\omega)$, bounded on the left by a semi-infinite layer with permittivity $\varepsilon_{1}(\omega)$ and permeability $\mu_{1}(\omega)$, and on the right by another semi-infinite layer with permittivity $\varepsilon_{3}(\omega)$ and permeability $\mu_{3}(\omega)$, where $\omega=2 \pi f$ is the angular frequency corresponding to frequency $f$. For now, all three layers are assumed to be linear and isotropic, but are free to exhibit loss in the form of complex material parameters. The system is excited by a plane wave at normal incidence, traveling in the positive $\hat{z}$ direction with angular frequency $\omega_{q}$, and originating from a source at $z=-\infty$. Without loss of generality, the polarization of the wave can be neglected. The one-dimensional wave equation in layer $i$ has the solution

$$
\mathcal{E}_{i}(z, t)=\operatorname{Re}\left\{E_{i}^{+} \exp \left(-\mathrm{i} \omega_{q} t\right)+E_{i}^{-} \exp \left(-\mathrm{i} \omega_{q} t\right)\right\}
$$

where $\mathcal{E}_{i}(z, t)$ is the real electric field and $E_{i}^{ \pm}$ $=A_{i}^{ \pm} \exp \left( \pm \mathrm{i} K_{i} z\right)$ is the complex amplitude of the electric field traveling in the $\pm \hat{z}$ directions. $K_{i}$ is the wave vector given by

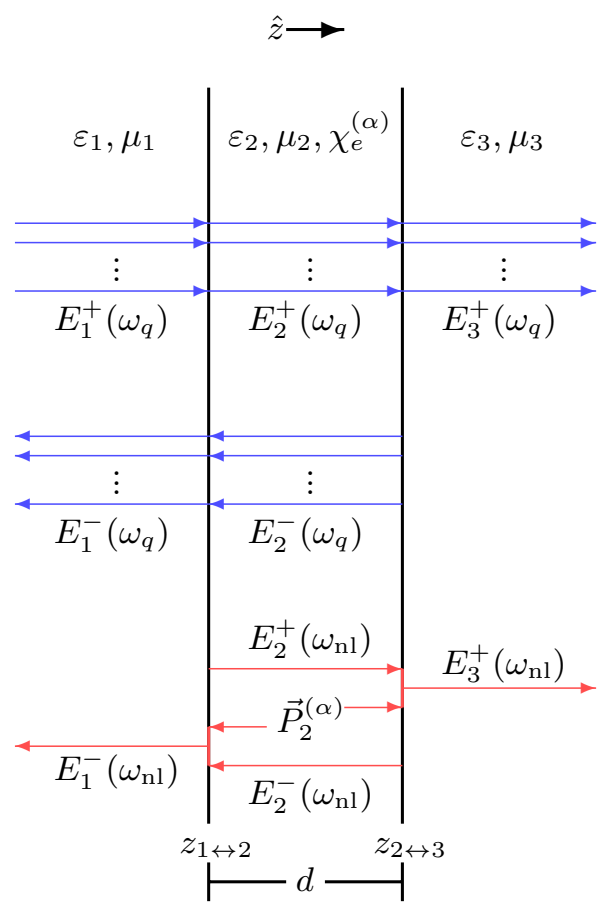

FIG. 1. (Color online) The system considered in this paper is composed of a central slab possessing an arbitrary electric nonlinearity of order $\alpha$, bounded by two semi-infinite linear media. Incident on this slab is an arbitrary number of normally propagating plane waves. The boundary conditions at the fundamental frequencies $\left(\omega_{q}\right)$ are presented on top (blue), while the boundary conditions at the nonlinear-generated frequency $\left(\omega_{\mathrm{nl}}\right)$ are shown below (red).

$$
K_{i}=n_{i} \frac{\omega_{q}}{c},
$$

where

$$
n_{i}=\frac{ \pm \sqrt{\varepsilon_{i} \mu_{i}}}{\sqrt{\varepsilon_{0} \mu_{0}}}
$$

is the index of refraction, $c$ is the speed of light in vacuum, and $\varepsilon_{0}$ and $\mu_{0}$ are the permittivity and permeability of free space, respectively. Note that the \pm in Eq. (3) allows for the possibility of negative refractive materials in the case where both $\varepsilon_{i}$ and $\mu_{i}$ are negative [24]. Thus, the three layers and two interfaces form a boundary value problem that can be solved by finding relations between the fields in the different regions. Writing the complex amplitudes in vector notation as

$$
\vec{E}_{i}(z)=\left[\begin{array}{c}
E_{i}^{+}(z) \\
E_{i}^{-}(z)
\end{array}\right],
$$

the fields on either side of the interface between the layers $i$ and $j$ at position $z_{i \leftrightarrow j}$ are related by

$$
\vec{E}_{j}\left(z_{i \leftrightarrow j}\right)=M_{i \rightarrow j} \vec{E}_{i}\left(z_{i \leftrightarrow j}\right) .
$$

In this last equation we have introduced the interface transfer matrix 


$$
M_{i \rightarrow j}=\frac{1}{t_{j \rightarrow i}}\left[\begin{array}{cc}
1 & r_{j \rightarrow i} \\
r_{j \rightarrow i} & 1
\end{array}\right],
$$

with the amplitude transmission and reflection coefficients of the interface given by

$$
t_{j \rightarrow i}=\frac{2 y_{j}}{y_{i}+y_{j}} \text { and } r_{j \rightarrow i}=\frac{y_{j}-y_{i}}{y_{i}+y_{j}},
$$

where $y_{i}=\sqrt{\varepsilon_{i} / \mu_{i}}$ is the admittance of medium $i$. Note that the interface transfer matrix, defined here in the positive direction, depends on the amplitude coefficients in the opposite direction. Similarly, the electric field at opposite ends of the same layer follows the relation

$$
\vec{E}_{j}\left(z_{i \leftrightarrow j}\right)=\Phi_{j} \vec{E}_{j}\left(z_{j \leftrightarrow k}\right)
$$

where $i, j$, and $k$ refer to consecutive layers,

$$
\Phi_{j}=\left[\begin{array}{cc}
\phi_{j} & 0 \\
0 & \phi_{j}^{-1}
\end{array}\right],
$$

is the propagation transfer matrix for layer $j, \phi_{j}=\exp ($ $\left.+\mathrm{i} K_{j} d_{j}\right)$ is the phase shift and attenuation factor from positive $\hat{z}$ propagation across layer $j$, and $d_{j}$ is the layer thickness.

The transfer matrix of a composite system is found by multiplying its individual transfer matrices in the appropriate order. Returning to the three-media example, we see that the composite matrix is

$$
M=\left[\begin{array}{ll}
M_{11} & M_{12} \\
M_{21} & M_{22}
\end{array}\right]=M_{2 \rightarrow 3} \Phi_{2} M_{1 \rightarrow 2} .
$$

Given that there is no negatively propagating field in the third layer, the fields incident on and exiting from the composite system are related by

$$
\left[\begin{array}{c}
E_{3}^{+}\left(z_{2 \leftrightarrow 3}\right) \\
0
\end{array}\right]=M\left[\begin{array}{l}
E_{1}^{+}\left(z_{1 \leftrightarrow 2}\right) \\
E_{1}^{-}\left(z_{1 \leftrightarrow 2}\right)
\end{array}\right],
$$

yielding amplitude reflection and transmission coefficients

$$
r=-\frac{M_{21}}{M_{22}} \text { and } t=\frac{\operatorname{det}[M]}{M_{22}},
$$

respectively. Assuming that the incoming wave amplitude $E_{1}^{+}$ is known, the fields in layer 2 at its interface with layer 1 are given by

$$
\vec{E}_{2}\left(z_{1 \leftrightarrow 2}\right)=M_{1 \rightarrow 2}\left[\begin{array}{l}
1 \\
r
\end{array}\right] E_{1}^{+}\left(z_{1 \leftrightarrow 2}\right) .
$$

If a total of $N$ waves at different frequencies are incident on the system, this procedure can be carried out independently for each frequency $\omega_{q}$ corresponding to $q=1,2, \ldots, N$, taking care to evaluate the transfer matrices appropriately.

\section{For an arbitrary nonlinear polarization}

As stated above, in the NDP limit, the presence of a nonlinearity in one or more layers is assumed to have a negligible effect on the incident waves, but will give rise to radiation at other frequencies. In this subsection, we derive the transmitted and reflected fields generated by an arbitrary higher-order polarization.

Let us consider an arbitrary higher-order polarization of order $\alpha$, generated by the medium in layer 2, at the angular frequency $\omega_{\mathrm{nl}}$. The presence of interfaces and reflections in the system leads to polarizations with multiple wave vectors at a single frequency. Thus, we introduce the decomposition by wave vector $Q$,

$$
P_{2}^{(\alpha)}\left(z, \omega_{\mathrm{nl}}\right)=\sum_{Q} P_{2}^{(\alpha, Q)}\left(z, \omega_{\mathrm{nl}}\right),
$$

such that the summation is over all existing wave vectors of the polarization at $\omega_{\mathrm{nl}}$ in layer 2. In examining an individual term of this summation, we note that while the phase distribution of the polarization and thus the electric field source is determined by the wave vector $Q$, the subsequent linear propagation of the fields generated by that source follow the appropriate wave vector $K_{2}=n_{2} \frac{\omega_{\mathrm{nl}}}{c}$. For clarity, we denote all terms involving the source distribution in layer 2 by the subscript $s$, while referring to the forward and backward propagating fields in the usual notation $\vec{E}_{2}\left(\omega_{n 1}\right)$. As shown in the Appendix, the electric field source produced by a higherorder polarization is given by

$$
E_{s}^{(Q)}\left(z, \omega_{\mathrm{nl}}\right)=\frac{P_{2}^{(\alpha, Q)}\left(z, \omega_{\mathrm{nl}}\right)}{\varepsilon_{s}^{(Q)}\left(\omega_{\mathrm{nl}}\right)-\varepsilon_{2}\left(\omega_{\mathrm{nl}}\right)},
$$

where

$$
\varepsilon_{s}^{(Q)}\left(\omega_{\mathrm{nl}}\right)=\frac{Q^{2}}{\omega_{\mathrm{nl}}^{2} \mu_{2}\left(\omega_{\mathrm{nl}}\right)} .
$$

It is important to emphasize the dependence of this electric field source on the specific wave vector of the nonlinear polarization, as this equation is, in part, a statement of the phase-matching condition. The interface transfer matrix for the electric field source is evaluated as

$$
M_{s \rightarrow 2}^{(Q)}=\frac{1}{t_{2 \rightarrow s}^{(Q)}}\left[\begin{array}{cc}
1 & r_{2 \rightarrow s}^{(Q)} \\
r_{2 \rightarrow s}^{(Q)} & 1
\end{array}\right],
$$

with reflection and transmission coefficients

$$
r_{2 \rightarrow s}^{(Q)}=\frac{\sqrt{\varepsilon_{2}}-\sqrt{\varepsilon_{s}^{(Q)}}}{\sqrt{\varepsilon_{s}^{(Q)}}+\sqrt{\varepsilon_{2}}} \text { and } t_{2 \rightarrow s}^{(Q)}=\frac{2 \sqrt{\varepsilon_{2}}}{\sqrt{\varepsilon_{s}^{(Q)}}+\sqrt{\varepsilon_{2}}} .
$$

Likewise, the propagation transfer matrix is given by

$$
\Phi_{s}^{(Q)}=\left[\begin{array}{cc}
\exp (+\mathrm{i} Q d) & 0 \\
0 & \exp (-\mathrm{i} Q d)
\end{array}\right] .
$$

Taking into account both the source and the propagating fields, the constraint of continuity across each interface leads to

$$
\vec{E}_{1}\left(z_{1 \leftrightarrow 2}, \omega_{\mathrm{nl}}\right)=M_{2 \rightarrow 1} \vec{E}_{2}\left(z_{1 \leftrightarrow 2}, \omega_{\mathrm{nl}}\right)+\sum_{Q} M_{s \rightarrow 1}^{(Q)} \vec{E}_{s}^{(Q)}\left(z_{1 \leftrightarrow 2}, \omega_{\mathrm{nl}}\right)
$$

and 


$$
\begin{aligned}
\vec{E}_{3}\left(z_{2 \leftrightarrow 3}, \omega_{\mathrm{nl}}\right)= & M_{2 \rightarrow 3} \Phi_{2} \vec{E}_{2}\left(z_{1 \leftrightarrow 2}, \omega_{\mathrm{nl}}\right) \\
& +\sum_{Q} M_{s \rightarrow 3}^{(Q)} \Phi_{s}^{(Q)} \vec{E}_{s}^{(Q)}\left(z_{1 \leftrightarrow 2}, \omega_{\mathrm{nl}}\right),
\end{aligned}
$$

where all transfer matrices must be evaluated at $\omega_{\mathrm{nl}}$, and we have used $M_{s \rightarrow 1}^{(Q)}=M_{s \rightarrow 2}^{(Q)} M_{2 \rightarrow 1}$ and $M_{s \rightarrow 3}^{(Q)}=M_{s \rightarrow 2}^{(Q)} M_{2 \rightarrow 3}$. Eliminating $\vec{E}_{2}$ from these equations and dropping the explicit $z$ and $\omega_{\mathrm{nl}}$ dependence gives

$$
\vec{E}_{3}=M_{2 \rightarrow 3} \Phi_{2}\left(M_{1 \rightarrow 2} \vec{E}_{1}+\vec{S}_{2}\right),
$$

where we have introduced the source term vector,

$$
\vec{S}_{2}=\sum_{Q}\left(\Phi_{2}^{-1} M_{s \rightarrow 2}^{(Q)} \Phi_{s}^{(Q)}-M_{s \rightarrow 2}^{(Q)}\right) \vec{E}_{s}^{(Q)} .
$$

Since there are no incident fields at $\omega_{\text {nl }}$, we can rewrite this as

$$
R\left[\begin{array}{c}
E_{3}^{+} \\
0
\end{array}\right]-L\left[\begin{array}{c}
0 \\
E_{1}^{-}
\end{array}\right]=\left[\begin{array}{ll}
R_{11} & -L_{12} \\
R_{21} & -L_{22}
\end{array}\right]\left[\begin{array}{c}
E_{3}^{+} \\
E_{1}^{-}
\end{array}\right]=\vec{S}_{2},
$$

where $R=\Phi_{2}^{-1} M_{2 \rightarrow 3}^{-1}$ and $L=M_{1 \rightarrow 2}$ are the transfer matrices on the right and left of the source term, respectively.

Thus, the total output fields at $\omega_{\mathrm{nl}}$ resulting from a nonlinearity of order $\alpha$ are given by the equation

$$
\left[\begin{array}{c}
E_{3}^{+}\left(\omega_{\mathrm{nl}}\right) \\
E_{1}^{-}\left(\omega_{\mathrm{nl}}\right)
\end{array}\right]=\left[\begin{array}{ll}
R_{11} & -L_{12} \\
R_{21} & -L_{22}
\end{array}\right]^{-1} \vec{S}_{2}\left(\omega_{\mathrm{nl}}\right) .
$$

It should be noted that in the limit of perfect phase matching, the magnitudes of the components of Eq. (23) inside and outside of the parentheses approach zero and infinity, respectively. However, their product has a finite value in this limit [22]. In a numerical realization of these equations, this singularity can be avoided by imposing a small mismatch in the relevant material parameters.

\section{B. Three-and four-wave mixing}

A host of nonlinear electromagnetic phenomena are understood in relation to a material's second and third-order nonlinear susceptibilities and, thus, the material's second and third-order polarizations [25]. The second-order interaction is carried out by the process of three-wave mixing of three waves satisfying the frequency-matching relation

$$
\omega_{q}+\omega_{r}=\omega_{q, r},
$$

where the subscripts $q$ and $r$ denote the contributing fundamental frequencies, and $\omega_{q, r}$ is the frequency of the generated wave. Note that the fundamental frequencies are free to be negative, as in the case of difference frequency generation (DFG), and degenerate, as in SHG. Similarly, the third-order process consists of four-wave mixing satisfying the relation

$$
\omega_{q}+\omega_{r}+\omega_{l}=\omega_{q, r, l} .
$$

All second- and third-order nonlinear phenomena can be described through these two processes. Furthermore, a given set of fundamental frequencies inside a second- or thirdorder nonlinear material will generate radiation at all frequency combinations satisfying the respective matching re- lation. In this section, we explicitly derive the nonlinear polarizations involved in the three- and four-wave mixing processes.

Since the formalism of nonlinear polarization is invariably messy, with multiple existing conventions, we will begin by defining some notation and relations. We consider a real electric field $\mathcal{E}(z, t)$ and polarization $\mathcal{P}(z, t)$, both of which can be transformed to the frequency domain with angular frequency $\omega_{q}$ and complex amplitudes $E\left(z, \omega_{q}\right)$ and $P\left(z, \omega_{q}\right)$, respectively. Dropping the explicit $z$ dependence, we define $E\left(-\omega_{q}\right) \equiv E\left(\omega_{q}\right)^{*}, P\left(-\omega_{q}\right) \equiv P\left(\omega_{q}\right)^{*}, \quad \omega_{-q} \equiv-\omega_{q}$, and $K_{-q} \equiv-K_{q}^{*}$, which leads to the relations

$$
\begin{gathered}
\mathcal{E}(t)=\mathcal{E}_{0}+\sum_{q} \frac{1}{2} E\left(\omega_{q}\right) \exp \left(-\mathrm{i} \omega_{q} t\right) \quad \text { and } \\
\mathcal{P}(t)=\mathcal{P}_{0}+\sum_{q} \frac{1}{2} P\left(\omega_{q}\right) \exp \left(-\mathrm{i} \omega_{q} t\right),
\end{gathered}
$$

where $\mathcal{E}_{0}$ and $\mathcal{P}_{0}$ are the zero-frequency (dc) amplitudes for the electric field and polarization, respectively. Both summations are over all values of $q \in\{-N, \ldots N\}$, excluding zero, where the magnitudes of $\mathcal{E}(t)$ and $\mathcal{P}(t)$ are split evenly between the positive and negative frequencies, resulting in the $\frac{1}{2}$ coefficient before each element. To make the dc terms compatible, the zero-frequency wave amplitudes in the following equations should be interpreted as $E(0)=2 \mathcal{E}_{0}$ and $P^{(\alpha)}(0)=2 \mathcal{P}_{0}$.

Starting from the formal definition of the second-order polarization and utilizing the permutation symmetry of the second-order nonlinear electric susceptibility $\chi_{e}^{(2)}\left(\omega_{q, r} ; \omega_{q}, \omega_{r}\right)$, we have

$$
\begin{aligned}
P^{(2)}\left(\omega_{q, r}\right) & =\frac{1}{2} \varepsilon_{0} \sum_{(q, r)} \chi_{e}^{(2)}\left(\omega_{q, r} ; \omega_{q}, \omega_{r}\right) E\left(\omega_{q}\right) E\left(\omega_{r}\right) \\
& =\frac{D}{2} \varepsilon_{0} \chi_{e}^{(2)}\left(\omega_{q, r} ; \omega_{q}, \omega_{r}\right) E\left(\omega_{q}\right) E\left(\omega_{r}\right),
\end{aligned}
$$

where the parentheses denote that the summation is over all possible permutations of the subscripts for a given value of $\omega_{q, r}$, and $D$ is the total number of permutations. With this last statement is the implicit assumption that only one nonlinear process contributes to a specific frequency of polarization. In the case of degeneracy, and in the NDP approximation, the contribution from each process can be calculated individually, and then summed, giving the total polarization at the degenerate frequency. Similarly, it can be shown that the third-order polarization term at $\omega_{q, r, l}$ is given by

$$
P^{(3)}\left(\omega_{q, r, l}\right)=\frac{D}{4} \varepsilon_{0} \chi_{e}^{(3)}\left(\omega_{q, r, l} ; \omega_{q}, \omega_{r}, \omega_{l}\right) E\left(\omega_{q}\right) E\left(\omega_{r}\right) E\left(\omega_{l}\right) .
$$

For convenience, the nonlinear susceptibilities will be referred to without explicit indication of their frequency dependence, but should not be assumed dispersionless.

The electric fields in each layer can be written in terms of both forward and backward propagating waves, which produce nonlinear polarizations that propagate with multiple 
wave vectors and corresponding amplitudes. For compatibility with the transfer matrix method, the polarizations must be separated by wave vector and represented in a vector form. Thus, we substitute into these last two expressions $E\left(\omega_{q}\right)$ $=A_{q}^{+} \exp \left(+\mathrm{i} K_{q} z\right)+A_{q}^{-} \exp \left(-\mathrm{i} K_{q} z\right)$, where $A_{q}^{ \pm}$is the complex amplitude of the wave propagating in the $\pm \hat{z}$-direction at frequency $\omega_{q}$. Note that here we are making an exception and using the subscript of the wave amplitude and wave vector to denote frequency and not layer. This results in a second-order polarization given by

$$
\vec{P}^{(2)}\left(\omega_{q, r}\right)=\vec{P}^{\left(2, K_{q}+K_{r}\right)}\left(\omega_{q, r}\right)+\vec{P}^{\left(2, K_{q}-K_{r}\right)}\left(\omega_{q, r}\right),
$$

where, in vector notation,

$$
\begin{aligned}
& \vec{P}^{\left(2, K_{q}+K_{r}\right)}\left(\omega_{q, r}\right)=\varepsilon_{0} \chi_{e}^{(2)} \frac{D}{2}\left[\begin{array}{l}
A_{q}^{+} A_{r}^{+} \exp \left[+\mathrm{i}\left(K_{q}+K_{r}\right) z\right] \\
A_{q}^{-} A_{r}^{-} \exp \left[-\mathrm{i}\left(K_{q}+K_{r}\right) z\right]
\end{array}\right], \\
& \vec{P}^{\left(2, K_{q}-K_{r}\right)}\left(\omega_{q, r}\right)=\varepsilon_{0} \chi_{e}^{(2)} \frac{D}{2}\left[\begin{array}{c}
A_{q}^{+} A_{r}^{-} \exp \left[+\mathrm{i}\left(K_{q}-K_{r}\right) z\right] \\
A_{q}^{-} A_{r}^{+} \exp \left[-\mathrm{i}\left(K_{q}-K_{r}\right) z\right]
\end{array}\right] .
\end{aligned}
$$

Similarly, the third-order polarization is found to be

$$
\begin{aligned}
\vec{P}^{(3)}\left(\omega_{q, r, l}\right)= & \vec{P}^{\left(3, K_{q}+K_{r}+K_{l}\right)}\left(\omega_{q, r, l}\right)+\vec{P}^{\left(3,-K_{q}+K_{r}+K_{l}\right)}\left(\omega_{q, r, l}\right) \\
& +\vec{P}^{\left(3, K_{q}-K_{r}+K_{l}\right)}\left(\omega_{q, r, l}\right)+\vec{P}^{\left(3, K_{q}+K_{r}-K_{l}\right)}\left(\omega_{q, r, l}\right),
\end{aligned}
$$

where

$$
\begin{aligned}
& \vec{P}^{\left(3, K_{q}+K_{r}+K_{l}\right)}\left(\omega_{q, r, l}\right) \\
& =\varepsilon_{0} \chi_{e}^{(3)} \frac{D}{4}\left[\begin{array}{l}
A_{q}^{+} A_{r}^{+} A_{l}^{+} \exp \left[+\mathrm{i}\left(K_{q}+K_{r}+K_{l}\right) z\right] \\
A_{q}^{-} A_{r}^{-} A_{l}^{-} \exp \left[-\mathrm{i}\left(K_{q}+K_{r}+K_{l}\right) z\right]
\end{array}\right], \\
& \vec{P}^{\left(3,\left(-K_{q}+K_{r}+K_{l}\right)\right)}\left(\omega_{q, r, l}\right) \\
& =\varepsilon_{0} \chi_{e}^{(3)} \frac{D}{4}\left[\begin{array}{l}
A_{q}^{-} A_{r}^{+} A_{l}^{+} \exp \left[+\mathrm{i}\left(-K_{q}+K_{r}+K_{l}\right) z\right] \\
A_{q}^{+} A_{r}^{-} A_{l}^{-} \exp \left[-\mathrm{i}\left(-K_{q}+K_{r}+K_{l}\right) z\right]
\end{array}\right], \\
& \vec{P}^{\left(3, K_{q}-K_{r}+K_{l}\right)}\left(\omega_{q, r, l}\right) \\
& =\varepsilon_{0} \chi_{e}^{(3)} \frac{D}{4}\left[\begin{array}{l}
A_{q}^{+} A_{r}^{-} A_{l}^{+} \exp \left[+\mathrm{i}\left(K_{q}-K_{r}+K_{l}\right) z\right] \\
A_{q}^{-} A_{r}^{+} A_{l}^{-} \exp \left[-\mathrm{i}\left(K_{q}-K_{r}+K_{l}\right) z\right]
\end{array}\right], \\
& \vec{P}^{\left(3, K_{q}+K_{r}-K_{l}\right)}\left(\omega_{q, r, l}\right) \\
& \quad=\varepsilon_{0} \chi_{e}^{(3)} \frac{D}{4}\left[\begin{array}{l}
A_{q}^{+} A_{r}^{+} A_{l}^{-} \exp \left[+\mathrm{i}\left(K_{q}+K_{r}-K_{l}\right) z\right] \\
A_{q}^{-} A_{r}^{-} A_{l}^{+} \exp \left[-\mathrm{i}\left(K_{q}+K_{r}-K_{l}\right) z\right]
\end{array}\right] .
\end{aligned}
$$

It should be noted that this matrix form is imprecise, in that the ordering of the terms in each individual matrix is not fixed but rather determined by the direction of energy propagation, such that, in each layer, the wave carrying energy in the $+\hat{z}(-\hat{z})$ direction is always on top (bottom). This can be handled by checking the sign of the quantity

$$
\beta^{(\alpha, Q)}=\frac{Q}{n^{(Q)}},
$$

where

$$
n^{(Q)}=\frac{ \pm \sqrt{\varepsilon_{s}^{(Q)} \mu_{2}}}{\sqrt{\varepsilon_{0} \mu_{0}}}
$$

is a pseudo-index of refraction describing the phase distribution of the nonlinear polarization. Correct propagation is ensured by reordering the vector elements such that $\beta^{(\alpha, Q)}$ is positive (negative) for the top (bottom) element. For example, if $\beta^{\left(3, K_{q}+K_{r}-K_{l}\right)}$ is negative, then the two terms in Eq. (37) must be flipped to ensure appropriate propagation. Mathematically, this can be accomplished by multiplying each vector term by the corrective matrix

$$
\frac{1}{2}\left[\begin{array}{cc}
1+\operatorname{sgn}\left(\beta^{(\alpha, Q)}\right) & 1-\operatorname{sgn}\left(\beta^{(\alpha, Q)}\right) \\
1-\operatorname{sgn}\left(\beta^{(\alpha, Q)}\right) & 1+\operatorname{sgn}\left(\beta^{(\alpha, Q)}\right)
\end{array}\right],
$$

where $\operatorname{sgn}(x)$ is the signum function.

We are now able to fully solve the forward problem of calculating the output fields generated by an arbitrary set of waves incident on a slab with a second-or third-order nonlinearity, in the NDP limit. In the context of our three-layer system, the decomposed polarizations in Eqs. (30) and (33) are evaluated from the fields given by Eq. (13). These, in turn, are used in Eqs. (15)-(25) to find the reflected and transmitted field amplitudes at each of the generated frequencies.

\section{Nonlinear parameter retrieval}

As stated in the introduction, our goal is to formulate a method for the homogenization of nonlinear metamaterials, extracting an effective nonlinear susceptibility from the results of simulation or experiment. However, the nonlinear susceptibility is only one of many factors determining the magnitude and phase of the generated fields. A homogenization method must normalize for these extraneous factors, isolating the value of the effective nonlinear susceptibility.

Let us return to our three-layer system, but under the assumption that the nonlinear susceptibility of the middle slab, $\chi_{e}^{(\alpha)}\left(\omega_{\mathrm{nl}} ; \ldots\right)$, is unknown, where $\left(\omega_{\mathrm{nl}} ; \ldots\right)$ represents the dependence of the nonlinear susceptibility on the generated and fundamental frequencies. On the other hand, we assume that the transmitted field at $\omega_{\mathrm{nl}}$ has been measured by experiment or simulation. For clarity, this field will be denoted by $E_{3, \text { exp }}^{+}\left(\omega_{\text {nl }}\right)$, where the subscript exp has been included to signify that this is a measured quantity.

From the equations of the previous section, we see that the nonlinear polarizations are directly proportional to the appropriate nonlinear susceptibility. This implies that the field sources, source terms, and generated fields are all directly proportional to the nonlinear susceptibility, as well. As such, the ratio of any of these quantities to the nonlinear susceptibility is, in fact, independent of the nonlinear susceptibility, and can be readily determined from the system's linear and dimensional properties. In particular, the ratio of the transmitted field to the nonlinear susceptibility, given by 
$E_{3}^{+}\left(\omega_{\mathrm{nl}}\right) / \chi_{e}^{(\alpha)}\left(\omega_{\mathrm{nl}} ; \ldots\right)$, can be calculated by another set of equations similar to those presented in sections 2.A and 2.B for the generated fields, but with the nonlinear susceptibility factored out in each case. Thus, we arrive at a procedure for determining the field-to-susceptibility ratio.

First, the linear and dimensional parameters of the system under consideration are determined by conventional methods. These are used in the equations of section 2.A.1 to find the fundamental fields in the nonlinear layer. Then, the ratios of the nonlinear polarization terms to the nonlinear susceptibility, $\vec{P}_{2}^{(\alpha, Q)} / \chi_{e}^{(\alpha)}$, are calculated simply by factoring the nonlinear susceptibility out of Eqs. (30)-(37). Similarly, we modify Eq. (15) to

$$
\vec{E}_{s}^{(Q)} / \chi_{e}^{(\alpha)}=\frac{\vec{P}_{2}^{(\alpha, Q)} / \chi_{e}^{(\alpha)}}{\varepsilon_{s}^{(Q)}-\varepsilon},
$$

and, in turn,

$$
\vec{S}_{2} / \chi_{e}^{(\alpha)}=\sum_{Q}\left(\Phi_{2}^{-1} M_{s \rightarrow 2}^{(Q)} \Phi_{s}^{(Q)}-M_{s \rightarrow 2}^{(Q)}\right) \vec{E}_{s}^{(Q)} / \chi_{e}^{(\alpha)},
$$

where we have dropped the explicit frequency dependence for convenience. This, at last, is used to calculate the fieldto-susceptibility ratios,

$$
\left[\begin{array}{c}
E_{3}^{+} / \chi_{e}^{(\alpha)} \\
E_{1}^{-} / \chi_{e}^{(\alpha)}
\end{array}\right]=\left[\begin{array}{ll}
R_{11} & -L_{12} \\
R_{21} & -L_{22}
\end{array}\right]^{-1} \vec{S}_{2} / \chi_{e}^{(\alpha)} .
$$

It is worth restating that these ratios are independent of the nonlinear susceptibility, but are otherwise calculated by the same procedure outlined at the end of Sec. II B.

We are now in a position to determine the nonlinear susceptibility of our sample. For consistency between our experimental and analytical results, we must have

$$
E_{3, \exp }^{+}\left(\omega_{\mathrm{nl}}\right)=\chi_{e}^{(\alpha)}\left(\omega_{\mathrm{nl}} ; \ldots\right) E_{3}^{+}\left(\omega_{\mathrm{nl}}\right) / \chi_{e}^{(\alpha)}\left(\omega_{\mathrm{nl}} ; \ldots\right) .
$$

Rearranging this statement, we arrive at our final retrieval equation,

$$
\chi_{e}^{(\alpha)}\left(\omega_{\mathrm{nl}} ; \ldots\right)=\frac{E_{3, \exp }^{+}\left(\omega_{\mathrm{nl}}\right)}{E_{3}^{+}\left(\omega_{\mathrm{nl}}\right) / \chi_{e}^{(\alpha)}\left(\omega_{\mathrm{nl}} ; \ldots\right)} .
$$

The numerator of Eq. (43) is determined directly from a nonlinear transmission experiment or simulation performed on the system, while the denominator is analytically derived from the system's linear and dimensional properties. Thus, Eqs. (39)-(43) constitute the generalized nonlinear susceptibility retrieval equations.

Numerically, the situation is even simpler, as a little thought reveals that computing the ratios of the various quantities to the nonlinear susceptibility is equivalent to calculating those same quantities with the substitution $\chi_{e}^{(\alpha)}$ =unity. This gives the alternative retrieval equation,

$$
\chi_{e}^{(\alpha)}\left(\omega_{\mathrm{nl}} ; \ldots\right)=\frac{E_{3, \exp }^{+}\left(\omega_{\mathrm{nl}}\right)}{\left.E_{3}^{+}\left(\omega_{\mathrm{n} 1}\right)\right|_{\chi_{e}^{(\alpha)}=1}},
$$

where the denominator can be calculated from the forward nonlinear transfer matrix equations, but with the aforementioned substitution for the nonlinear susceptibility. In addi- tion, it is clear that Eqs. (43) and (44) can be written in terms of the reflected amplitude $E_{1, \exp }^{-}\left(\omega_{\mathrm{nl}}\right)$ and its corresponding field-to-susceptibility ratio, if, alternatively, a reflection experiment is implemented.

\section{NUMERICAL VALIDATION}

The validity of the previous equations can be confirmed through comparison with an independent method of calculation. To this end, we have implemented time-domain finite element simulations using the COMSOL3.5a software package [26].

To test the second-order equations, we return to the threelayer system. We let $\varepsilon_{1}=\varepsilon_{3}=\varepsilon_{0}$ and $\mu_{1}=\mu_{3}=\mu_{0}$, so that the nonlinear slab is sandwiched by semi-infinite regions of vacuum. The nonlinear material is assigned a permittivity $\varepsilon_{2}=7 \varepsilon_{0}$, permeability $\mu_{2}=3 \mu_{0}$, and constant nonlinear susceptibility $\chi_{e}^{(2)}=10^{-12} \mathrm{~m} / \mathrm{V}$. The thickness of this material is varied over multiple simulations from 1 to $10 \mathrm{~mm}$. The input port generates a plane wave excitation according to $\mathcal{E}(t)$ $=\mathcal{E}_{1} \cos \left(\omega_{1} t\right)+\mathcal{E}_{2} \cos \left(\omega_{2} t\right)$, using the values $\mathcal{E}_{1}=10 \mathrm{GV} / \mathrm{m}$, $\mathcal{E}_{2}=7 \mathrm{GV} / \mathrm{m}, \omega_{1}=2 \pi \times 10 \mathrm{GHz}$, and $\omega_{2}=2 \pi \times 6 \mathrm{GHz}$. In order to avoid the transient effects and achieve an approximate steady-state solution, the sources are turned on for $1 \mathrm{~ns}$ before data is collected. This time-domain data is then Fourier transformed to obtain its spectrum amplitudes and phases.

The resulting field magnitudes for sum frequency (SFG), DFG, and SHG are compared in Fig. 2 for both approaches. The agreement between these two methods is excellent. Equally high agreement is also found between the computed phases (not shown).

The same procedure as above is implemented for a thirdorder nonlinear material, using a nonlinear susceptibility of $\chi_{e}^{(3)}=10^{-22} \mathrm{~m}^{2} / \mathrm{V}^{2}$. The incident radiation takes the form of $\mathcal{E}(t)=\mathcal{E}_{1} \cos \left(\omega_{1} t\right)+\mathcal{E}_{2} \cos \left(\omega_{2} t\right)+\mathcal{E}_{3} \cos \left(\omega_{3} t\right)$, with $\mathcal{E}_{1}$ $=10 \mathrm{GV} / \mathrm{m}, \quad \mathcal{E}_{2}=7 \mathrm{GV} / \mathrm{m}, \quad \mathcal{E}_{3}=5 \mathrm{GV} / \mathrm{m}, \quad \omega_{1}=2 \pi$ $\times 10 \mathrm{GHz}, \omega_{2}=2 \pi \times 6 \mathrm{GHz}$, and $\omega_{3}=2 \pi \times 9 \mathrm{GHz}$.

The results for four different frequency combinations are displayed in Fig. 3, once again showing excellent agreement between the two approaches despite considerable sensitivity to the thickness of the nonlinear slab. The transfer matrix method for nonlinear media is thus shown to be highly accurate in the NDP limit, especially with respect to the complicated contributions from the multiple reflections occurring at both the fundamental and the generated frequencies.

\section{APPLICATION TO EXPERIMENT}

It is highly desirable for a retrieval method to be experimentally practicable. In this section, we demonstrate the applicability of the transfer matrix retrieval by analyzing wave mixing measurements taken on a fabricated VLSRR medium.

We consider the same VLSRR metamaterial sample used by Huang et al. to study the power-dependent resonance frequency shift proportional to the third-order magnetic nonlinear susceptibility [17]. The unit cell is composed of a singly split copper ring on a $0.2 \mathrm{~mm}$ thick FR4 PCB substrate. The 


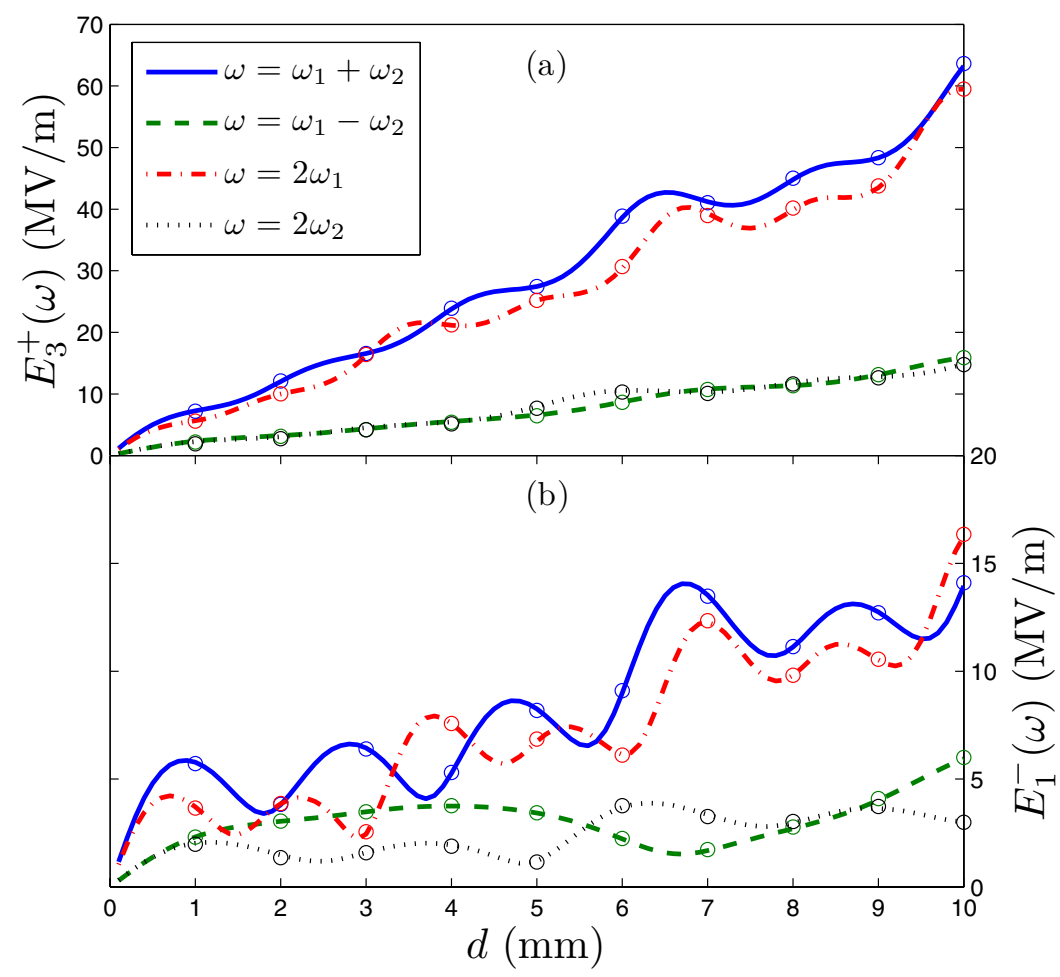

FIG. 2. (Color online) Comparison of the amplitudes of transmitted (a) and reflected (b) waves generated by three-wave mixing as calculated by the transfer matrix approach (lines) and finite element simulations (circles). ring is $17 \mu \mathrm{m}$ thick and $0.5 \mathrm{~mm}$ wide, with an inner radius of $4 \mathrm{~mm}$. The ring's gap is $1 \mathrm{~mm}$ across and loaded with a Skyworks SMV1231 varactor [27]. The varactor's capacitance is given by

$$
C\left(V_{D}\right)=C_{0}\left(1-V_{D} / V_{P}\right)^{M}
$$

where $V_{D}$ is the bias voltage, $C_{0}$ is the zero bias capacitance, $V_{P}=1.5 \mathrm{~V}$ is the intrinsic potential, and $M=0.8$ is the gradient coefficient.
A metamaterial slab is created by arranging multiple VLSRR cubic unit cells, $10 \mathrm{~mm}$ on a side, into a $3 \times 15$ $\times 1$ periodic structure. The rings are all oriented in the same direction so that the incident magnetic field will be along the SRR axes, while the direction of propagation is parallel to the sample's third dimension. This structure is placed in a transmission line optimized for TEM propagation below 2 $\mathrm{GHz}$, and shown via numerical simulations in CST Microwave Studio to exhibit a near-uniform concentration of $49 \%$

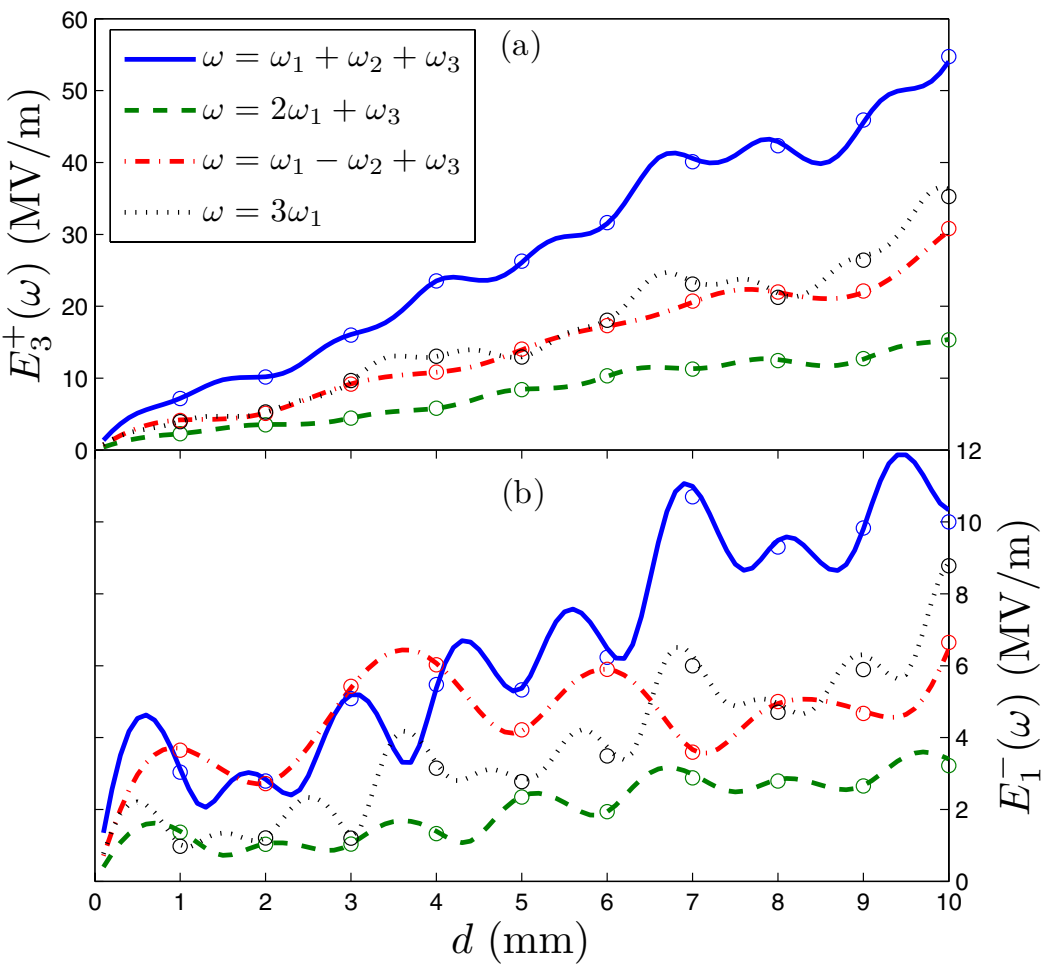

FIG. 3. (Color online) Comparison of the amplitudes of transmitted (a) and reflected (b) waves generated by four-wave mixing as calculated by the transfer matrix approach (solid lines) and finite element simulations (circles). 


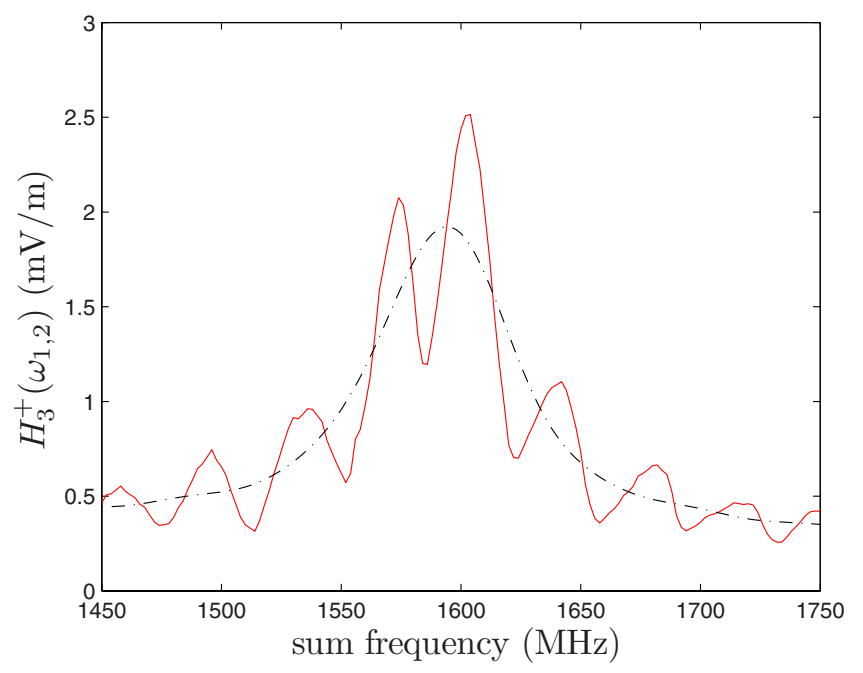

FIG. 4. (Color online) Plot of the transmitted SFG magnetic field spectrum. The red (solid) line is the raw data from experiment, while the black (dashed) line is the corresponding Fourier processed signal used in the retrieval.

of the incident power between the top and bottom plates [23]. The system is excited by Agilent N9310A and N5181A PSG vector signal generators operating at variable angular frequencies $\omega_{1}$ and $\omega_{2}$, respectively, each with an output power of $15 \mathrm{dBm}$. The harmonics created by the generators are eliminated by a Mini Circuits VLF-800+ filter. The transmission line and the connecting cables are calibrated with standard techniques. In accordance with these measurements, we find that the pump signal attenuates by $7.5 \pm .5 \mathrm{~dB}$ before the sample, and the sum frequency wave attenuates by $1.3 \pm .2 \mathrm{~dB}$ after the sample. The transmitted signals are measured using an Agilent E4404B PSA spectrum analyzer.

The linear response of the sample is measured and fitted using a Lorentz oscillator model, sufficiently described by permittivity

$$
\varepsilon(\omega)=1.63 \varepsilon_{0},
$$

and permeability

$$
\mu(\omega)=\mu_{0}\left(1+\frac{F \omega^{2}}{\omega_{0}^{2}-\mathrm{i} \gamma \omega-\omega^{2}}\right),
$$

where $F=0.142$ is the oscillator strength, $\omega_{0}=2 \pi$ $\times 813 \mathrm{MHz}$ is the angular resonance frequency, and $\gamma=2 \pi$ $\times 36 \mathrm{MHz}$ is the damping coefficient.

The frequency of the first signal generator is swept from $540 \mathrm{MHz}$ to $1000 \mathrm{MHz}$ at intervals of approximately $2 \mathrm{MHz}$, while the second signal generator operates at a constant frequency of $f_{2}=780 \mathrm{MHz}$. The sum frequency transmission data is shown in Fig. 4. The oscillations are a Fabry-Pérotlike resonance induced by reflections of the backward generated sum frequency wave off of the VLF-800+filter. These are removed via Fourier processing, resulting in the smoothed transmission spectrum shown against the raw data in Fig. 4.
For compatibility with our method, we can approximate the experimental setup as a $d=1 \mathrm{~cm}$ slab of homogeneous material, bounded by semi-infinite regions of vacuum, with linear properties given by Eqs. (46) and (47), and an unknown nonlinear susceptibility $\chi_{m}^{(2)}\left(\omega_{1,2} ; \omega_{1}, \omega_{2}\right)$. The magnitudes of the incident fields are given by

$$
\left|H_{1}^{+}\left(\omega_{1}\right)\right|=\left|H_{1}^{+}\left(\omega_{2}\right)\right|=\sqrt{\frac{2 I}{z_{0} S}},
$$

where $z_{0}=377 \Omega$ is the impedance of vacuum, $S=18 \mathrm{~cm}^{2}$ is the cross-sectional area of the waveguide, and $I$ is the input power of each signal generator corrected for the cable and waveguide losses. The nonlinearity in the VLSRR is magnetic in nature, originating from the relation between the inductive coupling of the copper ring and the nonlinear capacitance of the varactor-loaded gap. As stated earlier, the retrieval equations can be applied to a magnetic nonlinearity by replacing references to the electric field with the magnetizing field, the polarization with the magnetization, and swapping all occurrences of the permittivity and the permeability. Thus, we perform the nonlinear retrieval by evaluating the magnetic equivalent of Eq. (43), using the Fourier processed spectrum, corrected for cable losses, as an approximate measurement of $H_{3, \exp }^{+}\left(\omega_{1,2}\right)$. On the other hand, we calculate $H_{3}^{+}\left(\omega_{1,2}\right) / \chi_{m}^{(2)}\left(\omega_{1,2} ; \omega_{1}, \omega_{2}\right)$ according to Eqs. (39)-(41), using the second-order polarizations given by Eqs. (30)-(32). Despite the anisotropy of the sample, the method is still valid because only a single term of the permittivity, permeability, and susceptibility tensors is probed significantly. The experimental constraint of detecting the magnitude and not the phase of the outgoing wave means that, likewise, only the magnitude of the nonlinear susceptibility is retrieved. In addition, the uncertainty in the incident power results in an error of $\pm 15 \%$ in the retrieved susceptibility.

For comparison, we refer to the analytical expression recently presented by Poutrina et al. [28]. In this paper, the authors implement a perturbative solution of the nonlinear oscillator model to describe the effective $R L C$ circuit of the VLSRR medium. The resulting form of the nonlinear susceptibility is given in terms of the unit cell geometry and the varactor's intrinsic properties. This model was shown previously to quantitatively predict the second-order nonlinear susceptibility involved in SHG from this same VLSRR medium with high accuracy [23]. Poutrina et al.'s expression for the three-wave mixing nonlinear susceptibility is given by

$$
\chi_{m}^{(2)}\left(\omega_{1,2} ; \omega_{1}, \omega_{2}\right)=-\mathrm{i} a \frac{\omega_{0}^{4} \mu_{0} F A \omega_{1} \omega_{2} \omega_{1,2}}{D\left(\omega_{1}\right) D\left(\omega_{2}\right) D\left(\omega_{1,2}\right)},
$$

where $A$ is the area of the ring, $a=-M / 2 V_{p}$ is the secondorder coefficient in the perturbative expansion, $D(\omega)=\omega_{0}^{2}$ $-\mathrm{i} \gamma \omega-\omega^{2}$ is the denominator of the Lorentz oscillator at angular frequency $\omega$, and $F$ is the oscillator strength extracted from the previous linear retrieval. The magnitudes of the nonlinear susceptibility obtained by experimental retrieval and by Eq. (49) are both displayed in Fig. 5, showing excellent agreement. 


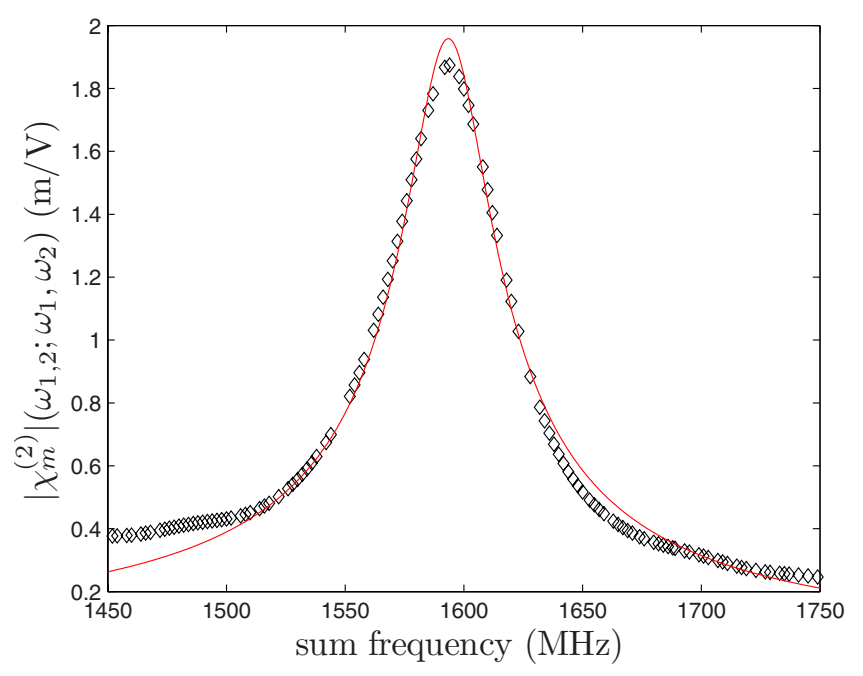

FIG. 5. (Color online) Comparison of the experimentally retrieved (black diamonds) and the theoretical (red line) second-order nonlinear susceptibility of the VLSRR medium.

\section{CONCLUSION}

We have demonstrated a generalized, transfer matrixbased nonlinear retrieval method that is exact in the limit of the NDP approximation. Furthermore, this method is proven highly applicable to metamaterial characterization, requiring only a transmission or reflection wave-mixing experiment to extract the effective nonlinear susceptibilities.

However, as with all metamaterial homogenization methods, the effective parameters are only valid when the wavelengths considered are much larger than the unit cell of the metamaterial. This can be a stricter limit for nonlinear phenomena, as the generated wavelengths may be several times smaller than those of the fundamental waves, as is the case in SFG and harmonic generation.

Due to the NDP approximation, this method is only valid for nonlinear phenomena in which the fundamental input waves are negligibly affected by the presence of the nonlinearity. As such, phenomena like the electro-optic effect and hysteresis, wherein the input wave is obviously and significantly altered, cannot be directly analyzed with this method. On the other hand, it should be possible to retrieve any nonlinear susceptibility whose generated frequency is not equal to one of its fundamentals by adjusting the input power to a level that satisfies the NDP approximation, notwithstanding the availability of appropriate power sources and detectors. A quick comparison of the magnitudes of the generated and fundamental waves implies that this approximation is reasonable for the presented experiment. Ideally, the validity of the retrieved nonlinear susceptibility can be rigorously confirmed by repeating the experiment over a wide range of input intensities. The results from this series of experiments should provide an NDP power threshold, below which the retrieved parameter is intensity independent and reliable.

In this retrieval, we assume that the second-order process is the only significant contributor to the SFG signal. However, all even higher-order terms also contribute. For high enough intensities of the fundamental waves, this retrieval method will return a second-order susceptibility that is intensity dependent, violating the usual power-series interpretation of susceptibility. This problem imposes a stricter constraint on the incident power than the NDP limit, but is also easily avoided by repeating the experiment for varying intensities of the fundamental waves.

In addition, we assumed an isotropic medium at the start of the analysis. However, careful control of the polarizations at the input and output allow this method to be used for anisotropic materials as long as only one term of the susceptibility tensor is being probed at a time. In this way, the entire tensor can be determined methodically, including the cross terms. It is often the case with metamaterials that only a small number of these terms are ultimately important. For non-normal incidence, on the other hand, a more complete formalism is necessary [29].

The original suspicion of nonlinear enhancement in metamaterials is confirmed by the remarkably large value of the nonlinear susceptibility retrieved from the VLSRR medium. Even when operated off resonance to avoid the large inherent losses, the nonlinear susceptibility is shown to be many orders of magnitude larger than that of naturally occurring materials. This opens the door for exciting applications, especially when considering the possibility of analogous metamaterials tuned to operate in the infrared and optical regimes.

\section{ACKNOWLEDGMENTS}

This work was supported by the Air Force Office of Scientific Research (Contract No. FA9550-09-1-0562). We thank Matthew S Reynolds for access to equipment and helpful discussions. S.L. thanks the Natural Sciences and Engineering Research Council of Canada for support.

\section{APPENDIX: GENERALIZED WAVE EQUATION FOR NONLINEAR MEDIA}

Presented here is the derivation of Eqs. (15) and (16).

In the absence of free charge and current, and assuming a steady-state solution proportional to $\exp (-\mathrm{i} \omega t)$, Maxwell's equations in layer $i$ are

$$
\begin{gathered}
\vec{\nabla} \times \vec{E}_{i}=\mathrm{i} \omega \vec{B}_{i}, \\
\vec{\nabla} \times \vec{H}_{i}=-\mathrm{i} \omega \vec{D}_{i}, \\
\vec{\nabla} \cdot \vec{D}_{i}=0, \\
\vec{\nabla} \cdot \vec{B}_{i}=0,
\end{gathered}
$$

while the material equations are

$$
\begin{gathered}
\vec{D}_{i}=\varepsilon_{i}(\omega) \vec{E}_{i}+\vec{P}_{i}^{(\alpha)}, \\
\vec{B}_{i}=\mu_{i}(\omega) \vec{H}_{i},
\end{gathered}
$$

where $E, D, B$, and $H$ are the complex electric, displacement, magnetic, and magnetizing fields, respectively, and $\vec{P}_{i}^{(\alpha)}$ is a 
polarization of order $\alpha>1$. We assume that the material parameters $\varepsilon_{i}$ and $\mu_{i}$ are time and space invariant so that they can be factored out of the curl and divergence operators.

Inserting Eq. (A6) in Eq. (A1) gives

$$
\vec{\nabla} \times \vec{E}_{i}=\mathrm{i} \omega \mu_{i}(\omega) \vec{H}_{i}
$$

Taking the curl of this equation and inserting Eqs. (A2) and (A5) results in

$$
\vec{\nabla} \times \vec{\nabla} \times \vec{E}_{i}=\omega^{2} \varepsilon_{i}(\omega) \mu_{i}(\omega) \vec{E}_{i}+\omega^{2} \mu_{i}(\omega) \vec{P}_{i}^{(\alpha)} .
$$

Using the identity

$$
\vec{\nabla} \times \vec{\nabla} \times \vec{E}_{i}=\vec{\nabla}\left(\vec{\nabla} \cdot \vec{E}_{i}\right)-\nabla^{2} \vec{E}_{i}
$$

and the fact that $\vec{\nabla} \cdot \vec{E}_{i}=0$, this can be rearranged to yield a wave equation according to

$$
\left[\nabla^{2}+\omega^{2} \varepsilon_{i}(\omega) \mu_{i}(\omega)\right] \vec{E}_{i}=-\omega^{2} \mu_{i}(\omega) \vec{P}_{i}^{(\alpha)} .
$$

Let us consider a polarization of the form $\vec{P}_{i}^{(\alpha)}$ $=\left|\vec{P}_{i}^{(\alpha)}\right| \hat{p} \exp [\mathrm{i}(Q z-\omega t)]$ and, similarly, an electric field $\vec{E}_{i}$ $=\left|\vec{E}_{i}\right| \hat{e} \exp [\mathrm{i}(Q z-\omega t)]$. Substituting these into Eq. (A10) gives

$$
\left[-Q^{2}+\omega^{2} \varepsilon_{i}(\omega) \mu_{i}(\omega)\right] \vec{E}_{i}=-\omega^{2} \mu_{i}(\omega) \vec{P}_{i}^{(\alpha)},
$$

or equivalently

$$
\vec{E}_{i}=\frac{\vec{P}_{i}^{(\alpha)}}{\varepsilon_{s}-\varepsilon_{i}(\omega)},
$$

where we have defined

$$
\varepsilon_{s}=\frac{Q^{2}}{\omega^{2} \mu_{i}(\omega)} .
$$

Note that, due to the dependence of $\varepsilon_{s}$ on $Q$, polarizations that propagate with multiple wave vectors at a single frequency, within the same layer, must be separated and handled individually.
[1] J. B. Pendry, A. J. Holden, D. J. Robbins, and W. J. Stewart, IEEE Trans. Microwave Theory Tech. 47, 2075 (1999).

[2] D. R. Smith, W. J. Padilla, D. C. Vier, S. C. Nemat-Nasser, and S. Schultz, Phys. Rev. Lett. 84, 4184 (2000).

[3] R. A. Shelby, D. R. Smith, and S. Schultz, Science 292, 77 (2001).

[4] A. A. Zharov, I. V. Shadrivov, and Y. S. Kivshar, Phys. Rev. Lett. 91, 037401 (2003).

[5] S. O'Brien, D. McPeake, S. A. Ramakrishna, and J. B. Pendry, Phys. Rev. B 69, 241101 (2004).

[6] M. W. Klein, C. Enkrich, M. Wegener, and S. Linden, Science 313, 502 (2006).

[7] M. W. Klein, M. Wegener, N. Feth, and S. Linden, Opt. Express $\mathbf{1 5}, 5238$ (2007).

[8] E. Kim, F. Wang, W. Wu, Z. Yu, and Y. R. Shen, Phys. Rev. B 78, 113102 (2008).

[9] E. Poutrina, S. Larouche, and D. R. Smith, Opt. Commun. 283, 1640 (2010).

[10] F. Niesler, N. Feth, S. Linden, J. Niegemann, J. Gieseler, K. Busch, and M. Wegener, (2009).

[11] I. V. Shadrivov, S. K. Morrison, and Y. S. Kivshar, Opt. Express 14, 9344 (2006).

[12] D. A. Powell, I. V. Shadrivov, Y. S. Kivshar, and M. V. Gorkunov, Appl. Phys. Lett. 91, 144107 (2007).

[13] I. V. Shadrivov, A. B. Kozyrev, D. W. van der Weide, and Y. S. Kivshar, Appl. Phys. Lett. 93, 161903 (2008).

[14] B. Wang, J. Zhou, T. Koschny, and C. M. Soukoulis, Opt.
Express 16, 16058 (2008).

[15] I. V. Shadrivov, A. B. Kozyrev, D. W. van der Weide, and Y. S. Kivshar, Opt. Express 16, 20266 (2008).

[16] D. A. Powell, I. V. Shadrivov, and Y. S. Kivshar, Appl. Phys. Lett. 95, 084102 (2009).

[17] D. Huang, E. Poutrina, and D. R. Smith, Appl. Phys. Lett. 96, 104104 (2010).

[18] D. R. Smith, S. Schultz, P. Markoš, and C. M. Soukoulis, Phys. Rev. B 65, 195104 (2002).

[19] X. Chen, T. M. Grzegorczyk, B.-I. Wu, J. Pacheco, and J. A. Kong, Phys. Rev. E 70, 016608 (2004).

[20] D. R. Smith, D. C. Vier, T. Koschny, and C. M. Soukoulis, Phys. Rev. E 71, 036617 (2005).

[21] S. Larouche and D. R. Smith, Opt. Commun. 283, 1621 (2010).

[22] D. S. Bethune, J. Opt. Soc. Am. B 6, 910 (1989).

[23] S. Larouche, A. Rose, E. Poutrina, D. Huang, and D. R. Smith, Appl. Phys. Lett. 97, 011109 (2010).

[24] V. G. Veselago, Sov. Phys. Usp. 10, 509 (1968).

[25] R. W. Boyd, Nonlinear Optics (Academic Press, New York, 2008).

[26] COMSOL, http://www.comsol.com

[27] Skyworks, Varactor SPICE models for RF VCO Applications, http://www.skyworksinc.com/uploads/documents/200315B.pdf

[28] E. Poutrina, D. Huang, and D. R. Smith, New J. Phys. 12, 093010 (2010).

[29] D. S. Bethune, J. Opt. Soc. Am. B 8, 367 (1991). 